\title{
Affinity depletion versus relative protein enrichment: a side-by-side comparison of two major strategies for increasing human cerebrospinal fluid proteome coverage
}

\author{
Eliska Jankovska ${ }^{1}$, Marek Svitek $^{2,3}$, Karel Holada $^{4}$ and Jiri Petrak ${ }^{1 *}$ (])
}

\begin{abstract}
Cerebrospinal fluid (CSF) is in direct contact with the central nervous system. This makes human CSF an attractive source of potential biomarkers for neurologic diseases. Similarly to blood plasma, proteomic analysis of CSF is complicated by a high dynamic range of individual protein concentrations and by the presence of several highly abundant proteins. To deal with the abundant human CSF proteins, methods developed for blood plasma/serum are routinely used. Multiple affinity removal systems and protein enrichment of less abundant proteins using a combinatorial peptide ligand library are among the most frequent approaches. However, their relative impact on CSF proteome coverage has never been evaluated side-by-side in a single study. Therefore, we explored the effect of CSF depletion using MARS 14 cartridge and ProteoMiner ligand library on the number of CSF proteins identified in subsequent LC-MS/MS analysis. LC-MS/MS analysis of crude (non-treated) CSF provided roughly 500 identified proteins. Depletion of CSF by MARS 14 cartridge increased the number of identifications to nearly 800, while treatment of CSF using ProteoMiner enabled identification of 600 proteins. To explore the potential losses of CSF proteins during the depletion process, we also analyzed the "waste" fractions generated by both methods, i.e., proteins retained by the MARS 14 cartridge, and the molecules present in the flow-through fraction from ProteoMiner. More than 250 proteins were bound to MARS 14 cartridge, 100 of those were not identified in the corresponding depleted CSF. Similarly, analysis of the waste fraction in ProteoMiner workflow provided almost 70 unique proteins not found in the CSF depleted by the ligand library. Both depletion strategies significantly increased the number of identified CSF proteins compared to crude CSF. However, MARS 14 depletion provided a markedly higher number of identified proteins (773) compared to ProteoMiner (611). Further, we showed that CSF proteins are lost due to co-depletion (MARS 14) or exclusion (ProteoMiner) during the depletion process. This suggests that the routinely discarded "waste" fractions contain proteins of potential interest and should be included in CSF biomarker studies.
\end{abstract}

Keywords: Cerebrospinal fluid, Depletion, CNS, Biomarkers, LC-MS/MS, Mass spectrometry

\footnotetext{
*Correspondence: jpetr@lf1.cuni.cz

${ }^{1}$ BIOCEV, First Faculty of Medicine, Charles University, Prague, Czech

Republic

Full list of author information is available at the end of the article
} 


\section{Introduction}

Proteomic analysis of human body fluids is a promising tool for the identification and detection of disease biomarkers. Human cerebrospinal fluid (CSF) is in direct contact with the central nervous system (CNS) and serves multiple functions including mechanic protection of the brain tissue, homeostasis maintenance, delivery of nutrients to the CNS, removal of waste and active regulation of CNS via hormones, neuropeptides, and other regulatory molecules. The composition of CSF thus reflects the physiological or pathological status of CNS and makes CSF an attractive source of potential biomarkers for neurologic diseases.

Abundances of several CSF proteins have been approved as diagnostic or prognostic markers in clinical practice. For instance, total tau protein concentration, phospho-tau concentration and presence of 42 amino acid form of $\beta$-amyloid are indicative of Alzheimer's disease [1]. Similarly, the appearance of oligoclonal immunoglobulin bands on electrophoretic gels of a CSF sample suggest the presence of multiple sclerosis [2]. However, for most diseases of the CNS, there are currently no reliable biomarkers with diagnostic, prognostic or therapeutic significance. New biomarkers for neurological diseases are therefore desirable.

CSF is formed partly $(\sim 20 \%)$ by active secretion of ependymal cells in the choroid plexuses of the brain ventricles but mostly by ultrafiltration of blood plasma by choroidal capillaries. CSF composition thus inevitably reflects the composition of blood plasma. However, the total protein concentration in CSF is 50-100 times lower, compared to plasma. While typical plasma protein concentration ranges roughly between 60 and $70 \mathrm{mg} / \mathrm{ml}$, normal CSF protein concentration is $0.2-0.7 \mathrm{mg} / \mathrm{ml}$ [3]. Proteomic analysis of CSF is, similarly to blood plasma, complicated by the complexity of its protein composition, by a very high dynamic range of concentrations of individual proteins and, most importantly, by the presence of several highly abundant proteins [4]. The ten most abundant CSF proteins (albumin, IgG, transthyretin, transferrin, $\alpha 1$-antitrypsin, apolipoprotein A, alpha-1-acid glycoprotein, haptoglobin, $\alpha 2$-macroglobulin, complement C3) are the same as in blood plasma and account for more than $80 \%$ of total CSF protein concentration. In CSF albumin alone accounts for $60 \%$ of total protein exceeding its relative abundance in plasma. On the other side, the relative abundance of immunoglobulins, namely IgG and IgM is significantly lower in CSF compared to plasma $[5,6]$. Furthermore, CSF contains specific high abundance proteins, for example, cystatin $\mathrm{C}$ and prostaglandin D2 synthase, both being synthesized in CNS [7].

High concentrations of the major proteins limit the depth of CSF proteomic analysis and at least partial removal of the most abundant proteins before LC-MS/ MS is needed to increase the coverage of CSF proteome [8]. Since there is no specific method for dealing with the abundant proteins in CSF, researchers have to resort to one of the methods developed for blood serum/plasma. Affinity depletion of the most abundant proteins and relative enrichment of medium and low abundance proteins using combinatorial peptide ligand library are among the most common.

Affinity depletion is based on the specific capture of target proteins using immobilized antibodies or other molecules (protein A/G, Cibacron Blue) with high affinity and specificity for the targets. Simultaneous removal of multiple high abundant proteins has become the major pre-analytical strategy for proteomic analysis of blood plasma [9]. Among the most used affinity depletion systems belong Multiple Affinity Removal Systems (MARS, Agilent) for depleting 2, 6, 7 or 14 most abundant proteins [10, 11], Seppro-IgY-14 (Sigma-Aldrich) and ProteoPrep 20 (Sigma-Aldrich) [12, 13] which remove 14 and 20 most abundant plasma proteins, respectively.

An alternative approach to the affinity depletion is the strategy of relative enrichment of low and medium abundance proteins based on their interactions with an immobilized combinatorial peptide ligand library, known under its market name ProteoMiner (Bio-Rad) [14]. Here, proteins from a complex sample bind to a vast spectrum of immobilized hexapeptide ligands through various types of interactions (ionic, hydrophobic interactions, hydrogen bonding, and van der Waal's force) with different affinities. The binding partners for each protein are present in limited numbers in the library; high-abundance proteins thus exceed the concentration of their binders, and their excess copies are washed off. Low-abundance proteins do not saturate their ligands and become relatively concentrated on the beads [15]. This approach is expected to enrich medium- and lowabundance proteins while relatively depleting or diluting the highly-abundant ones.

Both strategies, affinity depletion $[12,13]$ and relative enrichment by a ligand library $[16,17]$ have been shown to significantly increase CSF proteome coverage, but their relative effect has never been directly compared in a single study. Here, we evaluate the relative benefit of these two pre-analytical strategies side-by-side. Specifically, we evaluate the effect of Multiple Affinity Removal System for depletion of 14 most abundant proteins (MARS 14) and ProteoMiner ligand library on the number of proteins identified in human CSF using a standard LC-MS/ MS setup. Since the ultimate goal of both strategies is relative depletion of highly abundant proteins, we will use the term "depletion" for both throughout the manuscript. 


\section{Materials and methods Materials}

Acetonitrile (ACN) and ammonium bicarbonate (AMBIC) were purchased from Fluka (New Jersey, USA), Dulbecco's phosphate buffered saline (DPBS), sodium chloride, trifluoroacetic acid (TFA) and urea were from Sigma-Aldrich (St. Louis, USA), Bradford protein assay and iodoacetamide (IAA) were from Bio-Rad (Hercules, USA), dithiothreitol (DTT) and sequencing grade modified trypsin were purchased from Promega (Fitchburg, USA). Centrifugal filters $30 \mathrm{kDa}$ (spin concentrators) and water (Milli-Q) for all experiments were from Merck Millipore (Burlington, USA). Opti-Trap, desalting cartridge $(\mathrm{C} 18)$ was purchased from Optimize Technologies (Oregon City, USA). Cellulose acetate filters $0.22 \mu \mathrm{m}$ and 5 kDa MWCO (molecular weight cut-off) spin concentrator were purchased from Agilent Technologies (Santa Clara, USA).

\section{CSF collection}

The CSF samples were collected by lumbar puncture during spinal anesthetics procedures in patients of the Urology Clinic of General University Hospital undergoing minor surgery. The study was approved by the Ethics Committee of General University Hospital, Prague. CSF samples were centrifuged at $1500 \times g$ for $10 \mathrm{~min}$ at $4{ }^{\circ} \mathrm{C}$ within $30 \mathrm{~min}$ to remove cellular debris. Samples were then ultracentrifuged (Beckman Coulter Optima LE-80 K Ultracentrifuge, rotor SW 28, 120,000×g, $2 \mathrm{~h}$, $4{ }^{\circ} \mathrm{C}$ ) to remove all membranous vesicles and other particles. Obtained supernatants were aliquoted and stored at $-80^{\circ} \mathrm{C}$ until analysis.

\section{Preparation of pooled CSF sample}

Individual CSF samples were thawed on ice and protein concentration was determined using Bradford protein assay (Bio-Rad) at $595 \mathrm{~nm}$. A pooled CSF sample was generated from 5 patient samples. Each patient's sample contributed to the pool the same amount of protein. The pooled CSF sample was divided into aliquots, each representing $500 \mu \mathrm{g}$ of total protein. These aliquots were used throughout the study, always in technical triplicates.

\section{Multiple affinity depletion using MARS 14 cartridge}

Samples were processed in triplicates, according to manufacturer's instructions. MARS 14 (Agilent Technologies, Santa Clara, USA) cartridge was tempered at room temperature for $20 \mathrm{~min}$. Then the cartridge was equilibrated with $4 \mathrm{ml}$ of Buffer A. CSF sample $(500 \mu \mathrm{g})$ was diluted 1:1 in Buffer A and was filtered through $0.22 \mu \mathrm{m}$ filter $\left(10,000 \times g, 10 \mathrm{~min}, 4{ }^{\circ} \mathrm{C}\right)$. Since the volume capacity of the spin cartridge is limited the diluted CSF sample was loaded on the MARS 14 cartridge sequentially in several steps. After each centrifugation $(100 \times g, 1 \mathrm{~min})$ depleted CSF (flow through) was collected. The cartridge was then washed with $2 \times 400 \mu \mathrm{l}$ of Buffer A. Both washes were combined with the depleted CSF and concentrated using $5 \mathrm{kDa}$ MWCO spin concentrator to $200 \mu \mathrm{l}$. Proteins bound to the cartridge were eluted by $2.5 \mathrm{ml}$ of Buffer $\mathrm{B}$ as the "waste" fraction and stored at $-80{ }^{\circ} \mathrm{C}$ until analysis.

\section{Relative protein enrichment using ProteoMiner ligand library}

Protein enrichment was performed using Protein enrichment small-capacity kit (Bio-Rad, CA, USA) according to the manufacturer's instructions appropriately adapted to the low concentration of CSF. The kit is designed for $10 \mathrm{mg}$ of proteins. As our CSF samples contained $500 \mu \mathrm{g}$ of proteins, we used $25 \mu \mathrm{l}$ aliquots of beads for each CSF replicate. Beads were centrifuged $(1000 \times g, 1 \mathrm{~min}$, room temperature) to remove the storage liquid and then washed twice with $200 \mu \mathrm{l}$ of wash buffer. CSF sample containing $500 \mu \mathrm{g}$ of proteins was added to the beads and incubated for $2 \mathrm{~h}$ at room temperature in a 3D rotation mixer (RH-18, Hangzhou Miu Instruments). The sample was then centrifuged $(1000 \times g, 1 \mathrm{~min}$, room temperature) for $1 \mathrm{~min}$, and the flow-through fraction containing the unbound waste was collected. The beads with enriched proteins were washed twice with $200 \mu \mathrm{l}$ of wash buffer and once with $200 \mu \mathrm{l}$ of MiliQ water after $5 \mathrm{~min}$ incubation on the $3 \mathrm{D}$ rotation mixer, all three washes were joined with the waste fraction. Bound proteins were eluted from the beads in three steps each consisting of 15 min incubation in $20 \mu \mathrm{l}$ of elution reagent and centrifugation. The three eluate samples were combined representing the depleted CSF.

\section{Reduction, alkylation, digestion, and desalting}

Triplicates of CSF samples processed by both workflows and triplicates of crude CSF (representing $100 \mu \mathrm{g}$ of total protein each) were transferred to $30 \mathrm{kDa}$ cut off filters. The subsequent reduction, alkylation, and digestion of the samples were performed using the FASP (filteraided sample preparation) protocol [18]. Samples were alkylated for $20 \mathrm{~min}$ at room temperature in the dark by $100 \mu \mathrm{l}$ of iodoacetamide solution (50 mM iodoacetamide in $8 \mathrm{M}$ urea, $0.1 \mathrm{M}$ Tris $/ \mathrm{HCl} \mathrm{pH} \mathrm{8.5).} \mathrm{Samples} \mathrm{were} \mathrm{then}$ washed twice with $50 \mathrm{mM}$ ammonium bicarbonate, and proteomics grade trypsin was added. The samples were digested overnight at $37{ }^{\circ} \mathrm{C}$ and peptides were collected by centrifugation. The peptides were desalted on OptiPrep C-18 manually operated cartridge. The cartridge was equilibrated by $700 \mu \mathrm{l}$ of $80 \% \mathrm{ACN}$ in $0.1 \%$ TFA followed by $600 \mu \mathrm{l}$ of $0.1 \%$ TFA. The sample was loaded on the desalting cartridge and washed with $500 \mu \mathrm{l}$ of $0.1 \%$ 
TFA. Peptides were eluted by $200 \mu \mathrm{l}$ of $80 \%$ ACN in $0.1 \%$ TFA, dried in a speedvac (Eppendorf, Concentrator Plus) and stored at $-80^{\circ} \mathrm{C}$.

\section{nLC-MS ${ }^{2}$ analysis}

LC-MS/MS analyses were performed on a Thermo Orbitrap Fusion (Q-OT-qIT, Thermo) mass spectrometer equipped with Dionex Ultimate 3000 chromatograph. Samples were loaded onto the trap column (Acclaim PepMap300, C18, $5 \mu \mathrm{m}, 300 \AA$ A Wide Pore, $300 \mu \mathrm{m} \times 5 \mathrm{~mm}$, 5 Cartridges) for $4 \mathrm{~min}$ at $15 \mu \mathrm{l} / \mathrm{min}$ in $2 \%$ acetonitrile in $0.1 \%$ TFA. EASY-Spray column, $50 \mathrm{~cm} \times 75 \mu \mathrm{m}$ ID, PepMap C18, $2 \mu \mathrm{m}$ particles, $100 \AA$ pore size was used for the separation. Mobile phase A was composed of $2 \%$ acetonitrile in $0.1 \%$ formic acid. Mobile phase B was composed of $80 \%$ acetonitrile in $0.1 \%$ formic acid. The gradient was $90 \mathrm{~min}$ long at flow-rate $300 \mathrm{nl} / \mathrm{min}$ and temperature $55{ }^{\circ} \mathrm{C}$. Mobile phase $\mathrm{B}$ increases from 2 to $40 \% \mathrm{~B}$ at $60 \mathrm{~min}, 90 \% \mathrm{~B}$ at $61 \mathrm{~min}$, hold for $8 \mathrm{~min}$, and $2 \% \mathrm{~B}$ at $70 \mathrm{~min}$, hold for 15 min until the end of the run. Eluting peptide cations were converted to gas-phase ions by electrospray ionization and analyzed on a Thermo Orbitrap Fusion. Survey scans of peptide precursors from 400 to $1600 \mathrm{~m} / \mathrm{z}$ were performed at $120 \mathrm{~K}$ resolution (at $200 \mathrm{~m} / \mathrm{z}$ ) with a $5 \times 10^{5}$ ion count target. Tandem MS was performed by isolation at $1,5 \mathrm{Th}$ with the quadrupole, HCD fragmentation with a normalized collision energy of 30, and rapid scan MS analysis in the ion trap. The MS2 ion count target was set to $10^{4}$ and the max injection time was $35 \mathrm{~ms}$. Only those precursors with charge state 2-6 were sampled for MS2. The dynamic exclusion duration was set to $45 \mathrm{~s}$ with a $10 \mathrm{ppm}$ tolerance around the selected precursor and its isotopes. Monoisotopic precursor selection was turned on. The instrument was run in top speed mode with $2 \mathrm{~s}$ cycles.

\section{Data analysis}

The raw data obtained by LC-MS/MS were analyzed using MaxQuant software (version 1.6.0.1). The false discovery rate (FDR) was set to $1 \%$ for both, proteins and peptides. The minimum peptide length was set to 7 amino acids. The enzyme was set to trypsin with a maximum of two missed cleavages. Carbamidomethylation of cysteines was set as a fixed modification. N-terminal protein acetylation and methionine oxidation as variable modifications. Main search peptide tolerance was set to $4.5 \mathrm{ppm}$. MS/MS mass tolerance was set to $0.5 \mathrm{Da}$.

The Andromeda search engine was used for the MS/ MS spectra search against the UniProt Human database (downloaded in March 2018). The "match between runs" feature of MaxQuant was used to transfer identifications to other LC-MS/MS runs based on their masses and retention time (maximum deviation of
$0.7 \mathrm{~min}$ ). The "match between runs" was also used in quantification experiments. Quantifications were performed with the label-free algorithm in MaxQuant. Data analysis was performed using Perseus 1.6.1.3. software.

\section{Data presentation}

All Venn diagrams were created by the web application BioVenn [19].

\section{Results and discussion}

We evaluated the effect of the two major methods for depletion of abundant plasma proteins in CSF. Specifically, we assessed the impact of MARS 14 cartridge and ProteoMiner immobilized library on the number of proteins identified in triplicate CSF samples by a standard LC-MS/MS. In addition to the key fractions of interest, i.e., depleted CSF samples, we also determined the number of proteins present in the "waste" fractions, which are routinely discarded. (i.e., proteins retained on MARS 14 cartridge and proteins excluded by the ligand library and contained in the ProteoMiner flowthrough fraction). All samples originated from a single CSF sample pooled from 5 patients. A non-fractionated crude CSF sample was used as a reference; all samples were processed in technical triplicates. The experimental workflow is depicted in Fig. 1.

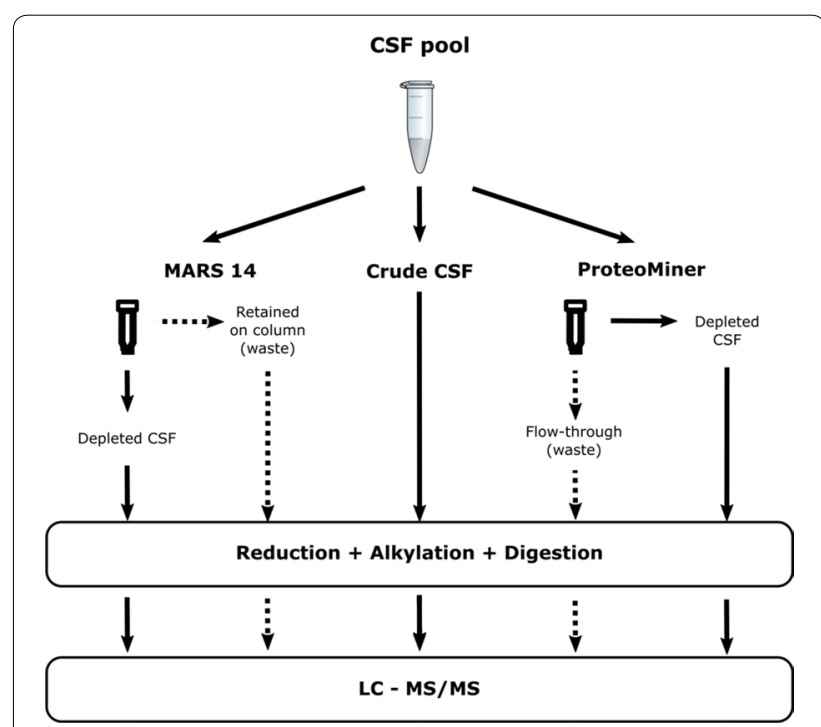

Fig. 1 Experimental workflow. Aliquots of a pooled human CSF sample were used. All analyses were performed in triplicates 
All proteins identified in the crude CSF, depleted CSF and corresponding waste fractions are listed in Additional file 1 .

\section{Crude CSF}

LC-MS/MS analysis of crude CSF triplicates provided 397, 419 and 427 identified proteins (Fig. 2). When the MS data of the triplicates were searched together, the analysis resulted in the identification of a total of 475 unique proteins, 275 proteins were present in all three replicates.

The number of identified proteins is with an agreement with a previous study using comparable LC-MS/MS setup [20]. The proteome coverage obtained for crude CSF was used as a reference for evaluation of the effect of CSF processing with MARS 14 cartridge and ProteoMiner library.

\section{CSF depleted by MARS 14 cartridge}

To evaluate the benefit of immunodepletion of major plasma proteins we used Multiple affinity removal cartridge for depletion of the 14 most abundant proteins of human plasma (MARS 14, Agilent), very often used in proteomic biomarker studies. Depletion of the most abundant CSF proteins using MARS 14 cartridge increased the number of identified proteins by $63 \%$ compared to crude CSF to total 773 in the triplicate analysis (Fig. 2). We identified between 633 and 696 proteins in the triplicate analyses. Of the total 773 identified proteins, 480 proteins were found in all three replicates.

\section{CSF depleted with ProteoMiner ligand library}

The principally different method of relative protein enrichment of medium and low abundance protein using
ProteoMiner hexapeptide ligand library increased the number of identified CSF proteins by $29 \%$ compared to crude CSF. The total number of unique proteins identified in the ProteoMiner-treated CSF triplicate was 611 (Fig. 2), 366 proteins were identified across all three replicates.

Considering the number of identified proteins, it is evident, that both depletion strategies are effective and significantly increase the number of identified proteins in comparison to crude CSF. However, MARS 14 immunodepletion enabled identification of a substantially more proteins.

\section{Depletion efficiency}

To evaluate the efficiency of depletion of the most abundant proteins we used label-free quantitative (LFQ) analysis. Relative depletion of the major proteins was calculated from the normalized intensities of peptide signals in the crude CSF and the depleted CSF. Results can be seen in Table 1 . The depletion efficiency of MARS 14 cartridge for the 14 most abundant (plasma) proteins was high, comparable with the efficiency claimed by the manufacturer and confirmed by others [10]. In general, CSF depletion with ProteoMiner decreased the amounts of the individual 14 most abundant proteins less effectively.

\section{The depleted CSF}

The two compared strategies employ radically different principles of dealing with the most abundant proteins, i.e., specific interactions with antibodies and other molecules versus less defined interactions with a library of peptide ligands. Also, MARS 14 cartridge retains unwanted proteins bound on the stationary phase, while

\section{Crude CSF}

475 proteins

(397)

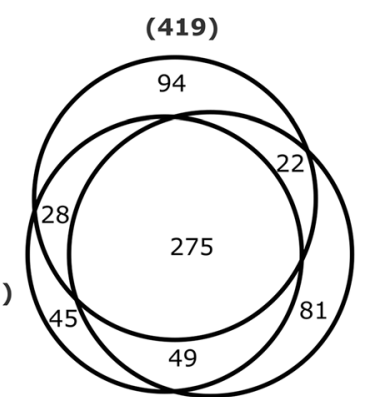

(427)

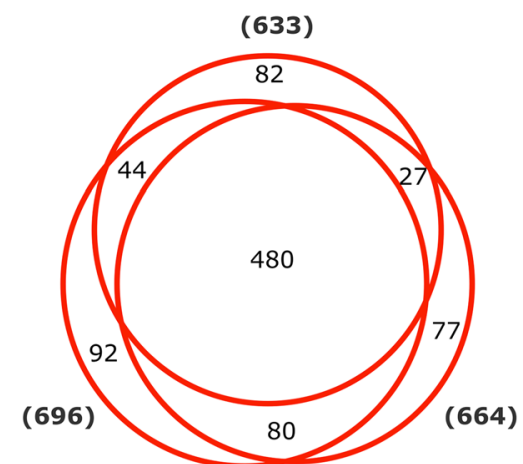

CSF depleted by ProteoMiner

611 proteins

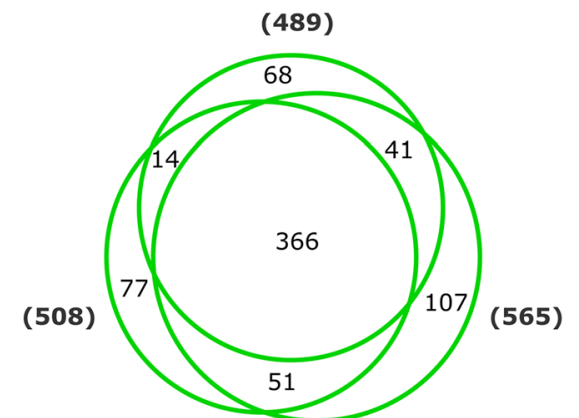

Fig. 2 CSF proteome coverage. Numbers of proteins identified by LC-MS/MS in the triplicate analyses of crude CSF, CSF depleted by MARS 14 and CSF depleted by ProteoMiner 
Table 1 The efficiency of depletion of the most abundant 14 plasma proteins by the MARS 14 immunodepletion and ProteoMiner

\begin{tabular}{|c|c|c|c|c|}
\hline & & & $\begin{array}{l}\text { MARS } 14 \\
\text { \%Removed }\end{array}$ & $\begin{array}{l}\text { ProteoMiner } \\
\text { \%Removed }\end{array}$ \\
\hline P02768 & Serum albumin & ALB & 97.4 & 71.9 \\
\hline P02647 & Apolipoprotein A-I & APOA1 & 99.9 & $<33$ \\
\hline P02652 & Apolipoprotein A-II & APOA2 & 75.6 & $<33$ \\
\hline P01876 & $\begin{array}{l}\text { Ig alpha-1 chain C } \\
\text { region }\end{array}$ & IGHA1 & 99.9 & 97.8 \\
\hline P01023 & $\begin{array}{l}\text { Alpha-2-macroglob- } \\
\text { ulin }\end{array}$ & $\mathrm{A} 2 \mathrm{M}$ & 99.9 & 68.4 \\
\hline P02763 & $\begin{array}{l}\text { Alpha-1-acid glyco- } \\
\text { protein } 1\end{array}$ & ORM1 & 99.7 & 97.8 \\
\hline P01009 & Alpha-1-antitrypsin & SERPINA1 & 99.7 & 84.3 \\
\hline P01857 & $\begin{array}{l}\text { Ig gamma-1 chain C } \\
\text { region }\end{array}$ & $|G H G|$ & 99.6 & 70.9 \\
\hline P02787 & Serotransferrin & $\mathrm{TF}$ & 99.4 & 96.1 \\
\hline P00738 & Haptoglobin & $H P$ & 98.7 & 92.1 \\
\hline P01024 & Complement C3 & C3 & 98.1 & $<33$ \\
\hline P02766 & Transthyretin & TTR & 95.2 & 89.0 \\
\hline P02675 & Fibrinogen beta chain & $F G B$ & 94.5 & $<33$ \\
\hline P02671 & $\begin{array}{l}\text { Fibrinogen alpha } \\
\text { chain }\end{array}$ & FGA & 93.7 & $<33$ \\
\hline P02679 & $\begin{array}{l}\text { Fibrinogen gamma } \\
\text { chain }\end{array}$ & FGG & 91.7 & $<33$ \\
\hline P01871 & $\lg M$ & IGHM & $100^{a}$ & $<33$ \\
\hline
\end{tabular}

Data from label-free quantification based on MS1 peptide signal intensities

a IgM was not detected in the CSF after MARS 14 immunodepletion

ProteoMiner excludes the abundant proteins in the mobile phase.

The principal differences in the two depletion strategies and their different depletion efficiencies may affect not only the number of identified CSF proteins but can also result in the identification of different sets of proteins. We compared the lists of proteins identified in CSF depleted by MARS 14 and ProteoMiner and confirmed that both methods provided a large number of unique proteins not identified by the other method (Fig. 3). The depletion of CSF using MARS 14 cartridge provided 336 such proteins not identified in the CSF treated by ProteoMiner and vice versa, the CSF sample treated with ProteoMiner provided 174 unique proteins not observed in the CSF depleted by MARS 14. This suggests that both methods introduce a bias toward and against some particular proteins.

We evaluated three basic properties of the identified proteins, namely MW, pI and hydrophobicity (GRAVY score) of all proteins identified in the depleted CSF samples. There was no systematic preference regarding MW, pI and hydrophobicity (GRAVY score) distributions between the two depleted fractions neither between the depleted samples and crude CSF (Additional file 2).

One example of depletion bias between these two methods is a higher number of various immunoglobulins found in the CSF sample depleted by ProteoMiner (33 identified proteins) compared to CSF treated by MARS 14 (10 proteins). In this case, it can be easily explained by the fact that MARS 14 specifically and efficiently targets and depletes immunoglobulins. However, among the method-specific proteins were also molecules previously identified as specific brain-enriched proteins [21] and therefore molecules with biomarker potential. For example, brain-enriched delta and Notch-like epidermal growth factor-related receptor (DNER_HUMAN), or Myelin-associated glycoprotein (MAG_HUMAN) were present only in the CSF depleted by MARS 14 while Protocadherin-8 (PCDH8_HUMAN) and Protocadherin gamma-C5 (PCDGM_HUMAN) were identified only in the CSF sample after ProteoMiner depletion [21]. In summary, both depletion methods have a profound beneficial impact on CSF proteome coverage. However, the lists of identified proteins differ between the two approaches suggesting a potential loss of biomarker proteins. To further explore the issue we analyzed the waste fractions generated in both workflows.

\section{The waste fractions}

Several studies reported that immunodepletion of highabundant proteins leads to an undesired co-depletion of numerous non-targeted proteins from the CSF $[12,13]$, including potential biomarkers of neurologic diseases [22]. Although we found no comparable study addressing the losses of CSF proteins in the ProteoMiner workflow, it can be expected that analogical losses occur, either due to low binding to the hexapeptide library or due to strong interactions with the abundant proteins that oversaturate their binding ligands.

To compare the extent of such unwanted protein losses due to co-depletion in both workflows, we analyzed the fraction of proteins retained by the MARS 14 cartridge and the equivalent proteins present in the flow-through fraction of the ProteoMiner library workflow. These waste fractions are routinely excluded from further analysis and discarded.

LC-MS/MS analysis of the waste fractions revealed significant extent of CSF protein loss in both depletion workflows. In addition to the most abundant CSF proteins (albumin, IgG, transthyretin, transferrin, $\alpha-1$ antitrypsin, haptoglobin $\alpha$, etc.), both waste fractions contained a substantial number of co-depleted non-target proteins. MARS 14 cartridge retained 214 proteins, and the ProteoMiner flow-through fraction contained 272 identified proteins (Fig. 4a). 


\section{CSF depleted by MARS 14}

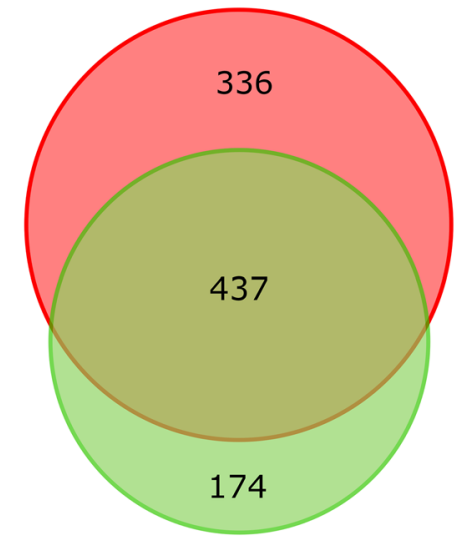

\section{CSF depleted by ProteoMiner}

Fig. 3 The unique and shared proteins identified in depleted CSF after depletion by MARS 14 or ProteoMiner. Over 330 proteins identified in CSF depleted by MARS 14 cartridge were not found in the CSF depleted by ProteoMiner library. Vice versa, 174 proteins were uniquely identified only in the CSF sample after ProteoMiner depletion

The significant number of the proteins identified in the waste fractions was also identified during the analysis of crude CSF. Large overlap with the crude CSF was found in the ProteoMiner waste (91\% identified proteins), while in the MARS 14 waste only $64 \%$ of identified proteins were also seen in the crude CSF (Fig. 4a). Although the numbers of the proteins identified in both waste fractions were roughly comparable (214 vs. 272$)$, the lists of the proteins differed significantly, only 111 proteins were present in both fractions. 103 and 161 proteins were specific for MARS 14 and ProteoMiner waste, respectively (Fig. 4b). Both these observations seem to reflect the fundamentally different fractionation principles of the depletion methods. However, again there was no systematic bias regarding MW, pI and hydrophobicity (GRAVY score) distributions between the two waste fractions or compared to depleted or crude CSF samples (see Additional file 2).

The high numbers of proteins identified in the waste fractions confirm the alarmingly large extent of the unwanted loss of proteins in both workflows. Importantly, many of the CSF proteins identified in the waste fractions were unique for the waste, i.e., were not found in the corresponding depleted CSF samples. We identified 115 and 69 such proteins in MARS 14 and ProteoMiner waste fractions, respectively (Fig. 5). Addition of the waste-specific proteins to the list of proteins identified in the corresponding depleted CSF increased the total coverage of CSF proteome roughly by $10-15$ percent to 888 and 680 identified proteins in MARS

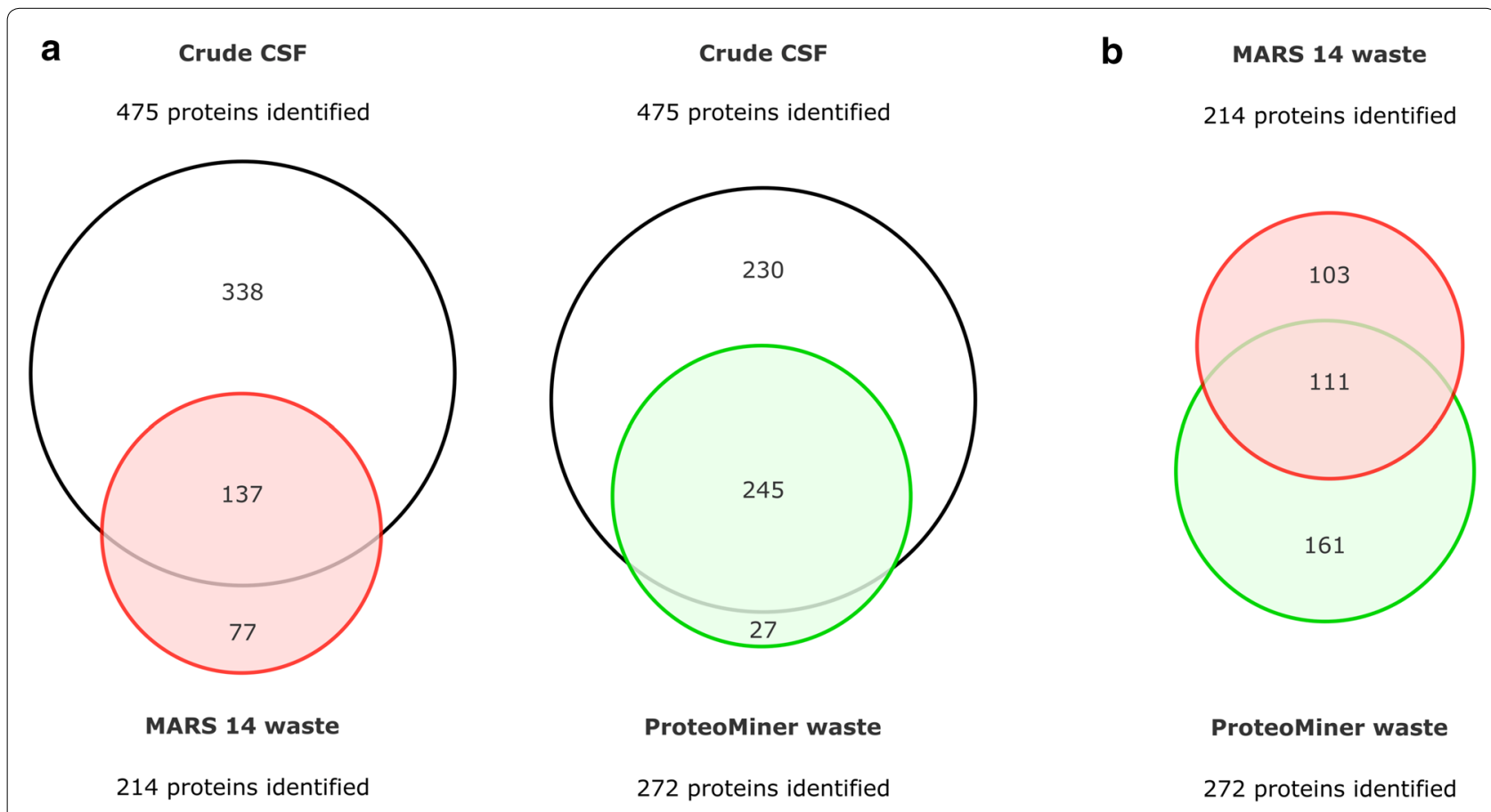

Fig. 4 Proteins identified in the waste fractions (proteins retained by MARS 14 cartridge and proteins excluded in the ProteoMiner flow-through). a The overlap of the proteins identified in the waste fractions with the proteins found in crude CSF. $\mathbf{b}$ The overlap between the two waste fractions 


\section{a CSF depleted by MARS 14}

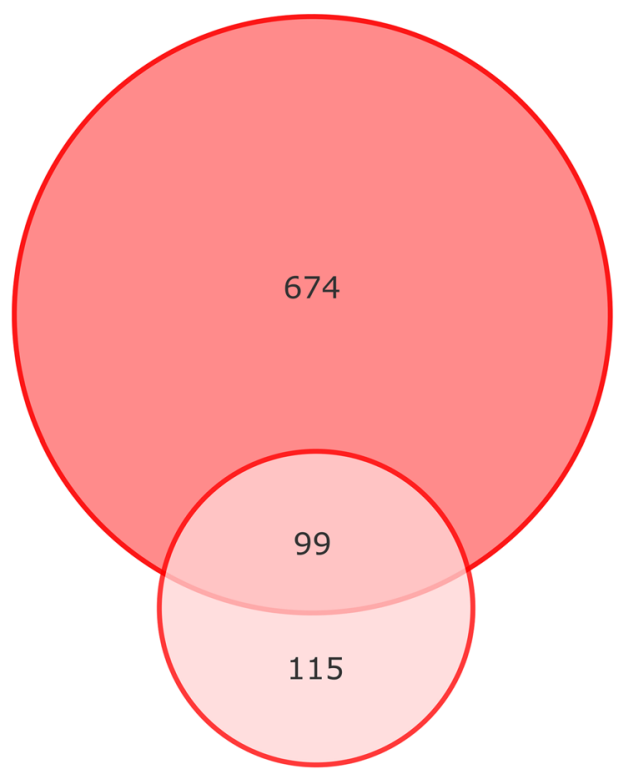

MARS 14 waste

\section{b CSF depleted by ProteoMiner}

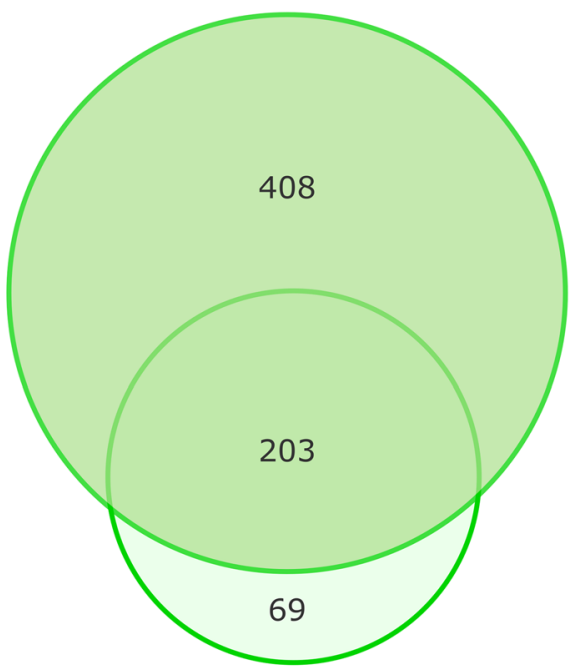

ProteoMiner waste

Total 888 proteins identified

Total 680 proteins identified

Fig. 5 The total number of proteins identified in the depleted CSF and corresponding waste fractions

14 and ProteoMiner workflow, respectively (Fig. 5). The strikingly high number of proteins lost during both depletion workflows may advocate future inclusion of the waste fractions into CSF biomarker studies. Such inclusion would be beneficial, especially if the waste fraction contained not only the major plasma constituents but also CNS-specific proteins. To evaluate the presence of such molecules, we compared the lists of proteins identified in the waste fractions with a list of previously characterized as brain-enriched proteins [21]. We found several such molecules to be present in the waste fractions, namely cell adhesion molecule 2 (CADM2_HUMAN), Amyloid-like protein 1 (APLP1_HUMAN) or Beta-Ala-His dipeptidase (CNDP1_HUMAN). Further search in literature identified additional waste-specific proteins with known relevance for neurophysiology and neuropathology. For example, MARS 14 cartridge fully retained Kinesinlike protein KIF1B (KIF1B_HUMAN) linked to Multiple Sclerosis susceptibility [23] and Tubulin beta-3 chain (TBB3_HUMAN) required for axon guidance [24]. Similarly, ProteoMiner waste fraction (but not CSF depleted by ProteoMiner) contained Proenkephalin-A (PENK_HUMAN) which has been considered as a potential marker of dementia and acute neuroinflammatory disorders in the form of its stable fragment (MR-PENK A) [25]. Proenkephalin-A was also described as an indicator of severity and clinical outcome in patients with ischemic stroke [26].

\section{Conclusions}

CSF is a valuable source of information on the status of CNS and a potential treasure trove of biomarkers. Compared to blood plasma, proteomic analysis of CSF is limited by its low protein concentration and the maximum volume of CSF that can be safely taken during the lumbar puncture. Considering also the potential health risks of the lumbar puncture, it is imperative to make proteomic analyses of CSF as efficient as possible. To maximize the number of identified CSF proteins we compared the benefits of two frequent, but principally different, methods for relative depletion of major plasma proteins-MARS 14 cartridge and ProteoMiner ligand library. To our knowledge, this is the first side-by-side comparison of these two major strategies in CSF. In our hands, both methods markedly improved CSF proteome coverage compared to analysis of crude (non-depleted) CSF. However, both methods enabled identification of exclusive 
sets of proteins in the resulting depleted CSF, reflecting the distinct principles of the fractionation.

The obvious beneficial effect of major protein depletion, however, comes for the price of unwanted protein loss during the procedure. Depletion or removal of any protein from a complex protein mixture under nondenaturing conditions inevitably leads to a co-depletion of other, non-targeted, proteins. In the case of MARS 14, it is either due to their direct interaction with the targeted proteins, due to cross-reactivity or a nonspecific interaction with the antibody, or via interactions with the stationary phase matrix. In the case of ProteoMiner, it may be due to insufficient interaction of proteins with the hexapeptide library or due to strong interactions with the excluded highly abundant proteins. In both tested methods, we observed a significant number of unique co-depleted proteins (proteins not identified in the corresponding depleted CSF samples), including brainenriched proteins, proteins with potential or confirmed roles in CNS pathologies-i.e., potential biomarkers. This clearly showed, that to maximize CSF proteome coverage and to limit the potential loss of biomarkers during the depletion, proteins retained by the MARS 14 cartridge (or excluded by ProteoMiner beads) should also be analyzed in future CSF biomarker studies. Alternatively, the addition of a crude CSF sample analysis may partially avoid the loss of some proteins due to co-depletion as the proteins contained in the waste fractions are largely a subset of crude CSF. However, in contrast to the waste fraction, the inclusion of crude CSF would require an additional volume of CSF.

Nevertheless, our main aim was to determine which of the two depletion methods enables higher coverage of the CSF proteome in an identical LC-MS/MS setup. MARS 14 cartridge enabled markedly higher proteome coverage with 773 identified proteins compared to ProteoMiner (611) and crude CSF (475). The superior performance of the MARS 14 cartridge can be further emphasized by quicker sample processing. Additionally, depletion of a single CSF sample using MARS 14 cartridge is less expensive, despite higher acquisition cost of MARS 14 cartridge.

To further increase the CSF coverage and make biomarker discovery more successful, novel strategies may be needed (in addition to a more extensive peptide fractionation). Among such tactics may be an orientation to specific forms of information carried by the CSF, namely neuropeptides or extracellular vesicles. These potential information carriers are usually excluded or lost in the standard proteomic analyses of CSF because of their low MW or sedimentation, respectively. Extracellular vesicles are cell-derived membrane structures carrying tissue-specific information and play a role in intercellular communication [27] and can be isolated from patient CSF and analyzed by LC-MS/MS [28]. Similarly, endogenous (neuro)peptides can be released from their carrier proteins, collected by ultrafiltration and analyzed [29]. Such semi-targeted approaches may reveal a brand new level of information carried by CSF.

\section{Additional files}

Additional file 1. A list of all identified proteins including crude CSF, depleted CSF and the waste fractions.

Additional file 2. Distributions of MW, pl and hydrophobicity (GRAVY score) in crude CSF, depleted CSF and the waste fractions.

Abbreviations

ACN: acetonitrile; AMBIC: ammonium bicarbonate; CNS: central nervous system; CSF: cerebrospinal fluid; DTT: dithiothreitol; IAA: iodoacetamide; LCMS/MS: liquid chromatography—tandem mass spectrometry; MARS: multiple affinity removal system; MW: molecular weight; pl: isoelectric point; TFA: trifluoracetic acid.

\section{Authors' contributions}

All authors read and approved the final version of the manuscript. E performed the CSF sample processing, LC-MS/MS analyses and wrote the manuscript. MS collected the CSF samples during spinal anaesthesia. $\mathrm{KH}$ conceived the study and interpreted the data. JP (the corresponding author) analysed and interpreted the data and wrote the manuscript. All authors read and approved the final manuscript.

\section{Author details}

${ }^{1}$ BIOCEV, First Faculty of Medicine, Charles University, Prague, Czech Republic. ${ }^{2}$ Department of Anesthesiology and Intensive Care, First Faculty of Medicine, Charles University, Prague, Czech Republic. ${ }^{3}$ General University Hospital, Prague, Czech Republic. ${ }^{4}$ Institute of Immunology and Microbiology, First Faculty of Medicine, Charles University, Prague, Czech Republic.

\section{Acknowledgements}

The study was supported by the Ministry of Health of the Czech Republic (NV 15-32961A), by the Ministry of Education, Youth and Sports of the Czech Republic (Progress Q26, NPU II-LQ1604, SW 260 374, UNCE/MED/016). Authors also acknowledge support by the projects CZ.1.05/2.1.00/19.0400 and CZ.1.05/1.1.00/02.0109 from the Research and Development for Innovations Operational Program (RDIOP) co-financed by European regional development fund and the state budget of the Czech Republic.

\section{Competing interests}

The authors declare that they have no competing interests.

\section{Availability of data and materials}

The dataset supporting the conclusions of this article (the complete list of proteins identified in the separate CSF fractions including accession numbers and sequence coverages) is included within the article as "Additional file 1". Raw MS data are available from the corresponding author upon request.

\section{Consent for publication}

Not applicable.

\section{Ethics approval and consent to participate}

The study was approved by the Ethics Committee of General University Hospital, Prague, Czech Republic. (Č.j. 120/14 Grant VES 2015 AZV 1. LF UK).

\section{Funding}

The study was supported by the Ministry of Health of the Czech Republic (NV 15-32961A), by the Ministry of Education, Youth and Sports of the Czech Republic (Progress Q26, NPU II-LQ1604, SW 260 374, UNCE/MED/016). 
Authors also acknowledge support by the projects CZ.1.05/2.1.00/19.0400 and CZ.1.05/1.1.00/02.0109 from the Research and Development for Innovations Operational Program (RDIOP) co-financed by European regional development fund and the state budget of the Czech Republic.

\section{Publisher's Note}

Springer Nature remains neutral with regard to jurisdictional claims in published maps and institutional affiliations.

Received: 17 December 2018 Accepted: 19 February 2019

Published online: 26 February 2019

\section{References}

1. Gozes I. Specific protein biomarker patterns for Alzheimer's disease: improved diagnostics in progress. EMPA J. 2017;8(3):255-9. https://doi. org/10.1007/s13167-017-0110-x.

2. McLean BN, Luxton RW, Thompson EJ. A study of immunoglobulin G in the cerebrospinal fluid of 1007 patients with suspected neurological diseases using isoelectric focusing and the log-lgG index. Brain. 1990:113:1269-89.

3. Yuan $X$, Desiderio DM. Proteomics analysis of human cerebrospinal fluid. J Chromatogr B Anal Technol Biomed Life Sci. 2005;815(1-2):179-89. https ://doi.org/10.1016/j.jchromb.2004.06.044.

4. Schutzer SE, et al. Establishing the proteome of normal human cerebrospinal fluid. PLoS ONE. 2010;5(6):1-7. https://doi.org/10.1371/journ al.pone.0010980.

5. Roche S, Gabelle A, Lehmann S. Clinical proteomics of the cerebrospinal fluid: towards the discovery of new biomarkers. Proteom Clin Appl. 2008;2(3):428-36. https://doi.org/10.1002/prca.200780040.

6. Fountoulakis $M$, et al. Depletion of the high-abundance plasma proteins. Amino Acids. 2004;27(3-4):249-59. https://doi.org/10.1007/s0072 6-004-0141-1.

7. Kroksveen AC, Opsahl JA, Aye TT, Ulvik RJ, Berven FS. Proteomics of human cerebrospinal fluid: discovery and verification of biomarker candidates in neurodegenerative diseases using quantitative proteomics. J Proteom. 2011;74(4):371-88. https://doi.org/10.1016/j.jprot.2010.11.010.

8. Wu C, Duan J, Liu T, Smith RD, Qian WJ. Contributions of immunoaffinity chromatography to deep proteome profiling of human biofluids. J Chromatogr B. 2016. https://doi.org/10.1016/j.jchromb.2016.01.015.

9. Yadav AK, et al. A systematic analysis of eluted fraction of plasma post immunoaffinity depletion: implications in biomarker discovery. PLoS ONE. 2011;6(9):1-9. https://doi.org/10.1371/journal.pone.0024442.

10. Tu Ch, et al. Depletion of abundant plasma proteins and limitations of plasma proteomics. J Proteome Res. 2010;9(10):4982-91. https://doi. org/10.1021/pr100646w.

11. Maccarrone $\mathrm{G}$, et al. Mining the human cerebrospinal fluid proteome by immunodepletion and shotgun mass spectrometry. Electrophoresis. 2004;25(14):2402-12. https://doi.org/10.1002/elps.200305909.

12. Fratantoni SA, Piersma SR, Jimenez CR. Comparison of the performance of two affinity depletion spin filters for quantitative proteomics of CSF: evaluation of sensitivity and reproducibility of CSF analysis using GeLCMS/MS and spectral counting. Proteom Clin Appl. 2010;4(6-7):613-7. https://doi.org/10.1002/prca.200900179.

13. Wetterhall M, Zuberovic A, Hanrieder J, Bergquist J. Assessment of the partitioning capacity of high abundant proteins in human cerebrospina fluid using affinity and immunoaffinity subtraction spin columns. J Chromatogr B Anal Technol Biomed Life Sci. 2010;878(19):1519-30. https://doi. org/10.1016/j.jchromb.2010.04.003.
14. Boschetti E, Righetti PG. The ProteoMiner in the proteomic arena: a non-depleting tool for discovering low-abundance species. J Proteom. 2008;71(3):255-64. https://doi.org/10.1016/j.jprot.2008.05.002.

15. Li L. Dynamic range compression with ProteoMiner ${ }^{\mathrm{TM}}$ : principles and examples. In: Posch A, editor. Proteomic profilling: methods and protocols. New York: Springer; 2015. p. 99-101.

16. Mouton-Barbosa E, et al. In-depth exploration of cerebrospinal fluid by combining peptide ligand library treatment and label-free protein quantification. Mol Cell Proteom. 2010;9(5):1006-21. https://doi.org/10.1074/ mcp.M900513-MCP200.

17. Sjödin MOD, Bergquist J, Wetterhall M. Mining ventricular cerebrospinal fluid from patients with traumatic brain injury using hexapeptide ligand libraries to search for trauma biomarkers. J Chromatogr B Anal Technol Biomed Life Sci. 2010;878(22):2003-12. https://doi.org/10.1016/j.jchro mb.2010.05.036.

18. Manza $\mathrm{LL}$, et al. Sample preparation and digestion for proteomic analyses using spin filters. Proteomics. 2005;5(7):1742-5. https://doi.org/10.1002/ pmic.200401063.

19. Hulsen T, de Vlieg J, Alkema W. BioVenn—a web application for the comparison and visualization of biological lists using areaproportional Venn diagrams. BMC Genome. 2008;9:1-6. https://doi. org/10.1186/1471-2164-9-488.

20. NunezGalindo A, Kussmann M, Dayon L. Proteomics of cerebrospinal fluid: throughput and robustness using a scalable automated analysis pipeline for biomarker discovery. Anal Chem. 2015;87(21):10755-61. https ://doi.org/10.1021/acs.analchem.5b02748

21. Begcevic I, Brinc D, Drabovich AP, Batruch I, Diamandis EP. Identification of brain-enriched proteins in the cerebrospinal fluid proteome by LC-MS/MS profiling and mining of the human protein atlas. Clin Proteom. 2016;13(1):1-13. https://doi.org/10.1186/s12014-016-9111-3.

22. Günther R, Krause E, Schümann M, Blasig IE, Haseloff RF. Depletion of highly abundant proteins from human cerebrospinal fluid: a cautionary note. Mol Neurodegener. 2015;10(1):53. https://doi.org/10.1186/s1302 4-015-0050-7.

23. Aulchenko YS, et al. Genetic variation in the KIF1B locus influences susceptibility to multiple sclerosis. Nat Genet. 2008;40(12):1402-3. https ://doi.org/10.1038/ng.251.

24. Tischfield MA, et al. Human TUBB3 mutations perturb microtubule dynamics, kinesin interactions, and axon guidance. Cell. 2010;140(1):7487. https://doi.org/10.1016/j.cell.2009.12.011.

25. Ernst A, et al. Midregional proenkephalin A and N-terminal Protachykinin $A$ are decreased in the cerebrospinal fluid of patients with dementia disorders and acute neuroinflammation. J Neuroimmunol. 2010;221(12):62-7. https://doi.org/10.1016/j.jneuroim.2010.02.004.

26. Doehner W, et al. Elevated plasma levels of neuropeptide proenkephalin a predict mortality and functional outcome in ischemic stroke. J Am Coll Cardiol. 2012;60(4):346-54. https://doi.org/10.1016/j.jacc.2012.04.024.

27. Chivet M, Hemming F, Pernet-Gallay K, Fraboulet S, Sadoul R. Emerging role of neuronal exosomes in the central nervous system. Front Physiol. 2012;3:145. https://doi.org/10.3389/fphys.2012.00145.

28. Chiasserini $\mathrm{D}$, et al. Proteomic analysis of cerebrospinal fluid extracellular vesicles: a comprehensive dataset. J Proteom. 2014;106:191-204. https:// doi.org/10.1016/j.jprot.2014.04.028.

29. Hölttä M, et al. An integrated workflow for multiplex CSF proteomics and peptidomics - identification of candidate cerebrospinal fluid biomarkers of Alzheimer's disease. J Proteome Res. 2015;14:654-63. https://doi. org/10.1021/pr501076j. 